Écrire

l'histoire

\section{Écrire l'histoire}

Histoire, Littérature, Esthétique

18 | 2018

Révolution

\title{
La Révolution tranquille et le Refus global
}

Sophie DuBoIs, Refus global. Histoire d'une réception partielle (2017)

\section{Étienne Beaulieu}

\section{(2) OpenEdition}

\section{Journals}

Édition électronique

URL : http://journals.openedition.org/elh/1628

DOI : $10.4000 /$ elh. 1628

ISSN : 2492-7457

Éditeur

CNRS Éditions

\section{Édition imprimée}

Date de publication : 20 novembre 2018

Pagination : 204-206

ISBN : 978-2-271-12431-9

ISSN : 1967-7499

Référence électronique

Étienne Beaulieu, « La Révolution tranquille et le Refus global », Écrire l'histoire [En ligne], 18 | 2018, mis en ligne le 20 novembre 2018, consulté le 16 février 2021. URL : http://journals.openedition.org/elh/ 1628 ; DOI : https://doi.org/10.4000/elh.1628

Ce document a été généré automatiquement le 16 février 2021.

Tous droits réservés 


\title{
La Révolution tranquille et le Refus global
}

Sophie DuBoIs, Refus global. Histoire d'une réception partielle (2017)

\author{
Étienne Beaulieu
}

\section{RÉFÉRENCE}

Sophie DuBoIs, Refus global. Histoire d'une réception partielle, Montréal, Presses de l'Université de Montréal (coll. « Nouvelles études québécoises »), 2017, 430 p.

1 La Révolution tranquille n'a rien d'une révolution ordinaire, puisqu'elle n'est justement pas révolutionnaire. Il n'y a pas eu dans cette transformation profonde de la société québécoise des années 1960 de mise à bas d'un pouvoir aristocratique quelconque, ou même d'une oligarchie financière ou sociale. Plutôt, une révolution dite "tranquille ", de la traduction anglo-canadienne quiet revolution d'où provient l'expression ayant servi d'abord à qualifier l'agitation politique et sociale qui gagne le Québec depuis la mort de Maurice Duplessis en 1959 et l'élection du gouvernement de Jean Lesage en 1960 (l'autoroute principale de la province, reliant Montréal et Québec, porte son nom), dont vont découler les grandes avancées de l'État-providence. Dans la foulée des mesures progressistes du gouvernement libéral d'Adélard Godbout (1939-1944), comme la création d'Hydro-Québec, le droit de vote accordé aux femmes, l'éducation obligatoire jusqu'à l'âge de quatorze ans et la gratuité scolaire au primaire, et malgré le retour en force de la très conservatrice Union nationale de Duplessis en 1944 jusqu'en 1959, dès l'élection de "l'équipe du tonnerre » de Jean Lesage en 1960, en quelques années, le Canada français se transformera en un Québec moderne qui s'affirme sur le plan linguistique, social et politique en effectuant une sortie de la religion toute en douceur malgré sa rapidité relative. Désormais, le Québec sera une société laïque, dans laquelle l'État et l'Église seront séparés, à l'image de la loi Combes de 1905. À partir de ce point tournant de l'histoire québécoise, une révolution semblable à celle des Trente Glorieuses s'accomplit en raccourci, à la différence notable d'une forte connotation 
nationaliste de la Révolution tranquille qui donne aux francophones un accès égalitaire aux postes clés de l'économie et de la politique (« Maîtres chez nous », dit le slogan de la campagne de 1962). Une libéralisation des mœurs analogue à celle de Mai 68 s'empare du Québec sous le pouvoir montant de la jeunesse d'une génération née dans l'après-guerre, comme partout du côté des vainqueurs occidentaux, que l'on qualifie désormais de baby-boom, jusqu'à devenir aujourd'hui, soixante-dix ans plus tard, le papy-boom, alors que la pyramide des âges se voit inversée au point où la majorité de la population québécoise se trouve dans cette tranche d'âge, quand c'était l'inverse dans les années 1960, à l'époque que l'essayiste François Ricard a nommée «la génération lyrique ${ }^{1} »$.

2 Lorsque l'on cherche les premiers symptômes de cette révolution étrange, à la fois fulgurante et radicale, mais pourtant très douce et clémente, il est fréquent de se tourner vers le Refus global, cet événement de société aujourd'hui devenu mythique et ressassé jusqu'à plus soif. Résumons les faits : en 1948, le peintre Paul-Émile Borduas, alors professeur à l'École du meuble de Montréal, publie en pleine époque duplessiste, surnommée «la Grande Noirceur », un texte manifeste éponyme « Refus global » dans un recueil de textes artistiques colligé et publié sous le titre, maintenant célébrissime, de Refus global. Ce recueil comprend trois textes de Paul-Émile Borduas, trois du poète Claude Gauvreau, un du poète, dramaturge et psychiatre Bruno Cormier, un de la sculptrice, chorégraphe et danseuse Françoise Sullivan, puis un dernier du peintre Fernand Leduc. Le texte est accompagné d'illustrations et de photographies, notamment du peintre Jean-Paul Riopelle. Le recueil est cosigné par quinze personnalités artistiques et publié aux éditions Mythra-Mythe à quatre cents exemplaires (qui se vendent aujourd'hui à plusieurs dizaines de milliers de dollars pièce, soit à l'encan, soit dans le réseau des librairies indépendantes). Le texte éponyme de Borduas lui-même, personnalité alors plus en vue que les plus jeunes signataires du recueil, critique sévèrement la société canadienne-française pour son immobilisme social et pour son repli au sein de l'Église catholique. L'influence du surréalisme de Breton est manifeste dans ce texte, mais aussi dans l'ensemble du recueil ; pour autant, celui-ci ne saurait s'y réduire, comme on le fait souvent en évoquant l'aventure artistique de ceux qu'on appelle depuis «les Automatistes", en prenant cet épisode comme point de départ de la Révolution tranquille.

Or, comme le montre Sophie Dubois dans cette étude très fouillée de la réception, ou plutôt de la non-réception de cette publication maintenant trop connue et aujourd'hui presque introuvable en édition originale, la Révolution tranquille s'est élaborée dans le mythe du Refus global en omettant de lire vraiment ce recueil multidisciplinaire. Plusieurs obstacles ont opacifié la lecture de ces pages maintenant trop célèbres pour être réellement prises en compte avec soin et attention. D'abord, la réduction du recueil au seul texte manifeste de Paul-Émile Borduas, alors plus âgé que les autres auteurs ou signataires et dont le congédiement dans les suites de cette publication a contribué à rendre légendaires les pages de son seul manifeste et à occulter tout le reste du recueil, Refus global, aujourd'hui à peine connu du grand public en comparaison du texte de Borduas, "Refus global ». Même les autres textes de Borduas, pourtant publiés dans le même recueil, souffrent de cette disproportion d'attention, comme c'était d'ailleurs le cas dès les premières années de réception, le phénomène s'amplifiant ensuite jusqu'à ne plus exister qu'en texte solitaire dans les anthologies de la littérature québécoise. Ensuite, la dimension interdisciplinaire du recueil a rendu la 
lecture de Refus global très partielle, les arts visuels, la littérature, la sociologie et l'histoire se bornant à leur propre champ, sans tenir compte de la proposition justement globale du recueil, qui se voulait un appel sans frontière artistique ou sociale, à l'époque où, comme le rappelle Dubois en s'inspirant très fortement du sociologue Pierre Bourdieu, l'autonomisation de la littérature québécoise s'effectuait un siècle après la France. Et c'est d'ailleurs l'un des enjeux importants du recueil Refus global qui est demeuré passablement occulté tout au long de sa (non-)réception : celui du rattrapage culturel à l'égard de la France, pourtant abordé explicitement par au moins Gauvreau et Riopelle dans leurs textes passés presque sous silence dans toute l'histoire de la réception de Refus global.

4 L'intérêt majeur de l'étude de Dubois réside toutefois surtout dans la mise en évidence de la puissance de lecture des écrivains, en particulier des essayistes, de la Révolution tranquille qui s'approprient rétrospectivement l'événement de Refus global comme une percée de leurs propres avancées, souvent des décennies plus tard. Après les textes de Gauvreau, qui fondent le mythe Borduas dès son congédiement de l'École du meuble, ceux de Pierre Vadeboncœur, notamment La Ligne du risque, contribuent fortement à créer le récit commun de l'avènement du Québec à la modernité dès le clairon qu'auraient sonné Refus global et la personne même de Paul-Émile Borduas. Une quantité impressionnante de textes suivront cette doxa jusqu'à créer un pont interprétatif entre Refus global et la Révolution tranquille, l'un légitimant l'autre au point de s'y confondre souvent. Dubois qualifie ces textes de " verrous interprétatifs ", puisqu'ils empêchent une lecture du recueil Refus global et qu'ils réduisent, comme presque toujours, l'événement à un seul texte et à un seul homme, montré comme héroïque résistant face à un système politique tout-puissant, le duplessisme, dont il aurait été la victime exemplaire sacrifiée sur l'autel de la nation. Autrement dit, la Révolution tranquille s'est elle-même constituée en mythe en créant celui du Refus global de Borduas, dans un rare cas de réception hyper-créatrice.

Maintenant, à tout prendre, pour que s'effectuent des changements de société majeurs comme ceux des années 1960 au Québec, une nation n'a-t-elle pas besoin de mythes fondateurs afin que s'y trouve l'énergie nécessaire à l'épanouissement des vœux d'une collectivité ? C'est malheureusement un aspect important de la question qui reste informulé dans cette étude pourtant riche et très novatrice.

\section{NOTES}

1. François RICARD, La Génération lyrique. Essai sur les premiers-nés du baby-boom, Montréal, Boréal, 1992. 
INDEX

oeuvrecitee Refus global. Histoire d'une réception partielle - (Sophie Dubois, 2017)

\section{AUTEURS \\ ÉTIENNE BEAULIEU}

Écrivain, professeur et éditeur, Étienne Beaulieu dirige les éditions Nota bene, enseigne la littérature au Cégep de Drummondville et dirige la programmation des Correspondances d'Eastman. Il a fait paraître un récit qui lui a valu quelques prix (Trop de lumière pour Samuel Gaska, Lévesque, 2014, prix Jacques-Cartier [Lyon-Montréal], prix Alfred-Desrochers, Grand Prix de la Ville de Sherbrooke) et qui sera suivi en 2018 par un récit épique, Thomas Aubert. La pensée et la mer. Il a fait paraître aussi des essais littéraires en recueils (Splendeur au bois Beckett, Nota bene, 2016 ; L'Âme littéraire, Nota bene, 2014 ; et Sang et Lumière. La communauté du sacré dans le cinéma québécois, L'Instant même, 2007). Du côté plus académique, il a publié sa thèse, La Fatigue romanesque de Joseph Joubert (1754-1824), aux Presses de l'université Laval en 2007, et un recueil d'études est à paraître sur le romantisme français, L'Éclat du neutre. Études sur les cultures romantiques de la prose (Paris, Classiques Garnier). Il est aussi cofondateur des cahiers littéraires Contre-jour et membre de la rédaction depuis 2002. 